\title{
ACTH gel in the treatment of multiple sclerosis exacerbation: a case study
}

This article was published in the following Dove Press journal:

International Medical Case Reports Journal

7 January 2015

Number of times this article has been viewed

\section{Salvatore Napoli}

Neuro Institute of New England, Foxborough, MA, USA
Correspondence: Salvatore Napoli Neuro Institute of New England, I5 Payson Road, Foxborough, Massachusetts 02035, USA

$\mathrm{Tel}+\mid$ 78I 55I 58I2

Fax + I 508698 867|

Email sqnapoli@gmail.com
Abstract: Patients may refuse, be unable to use, or show nonresponse to conventional steroid treatment of multiple sclerosis (MS) exacerbation. Adrenocorticotropic hormone (ACTH), one of several melanocortin peptides with mechanisms of action beyond steroidogenesis, should be reconsidered in the treatment of MS exacerbations. The current case report presents the treatment outcome of a patient with new-onset MS exacerbation treated with ACTH following lack of response to steroid treatment. A 49-year-old female presented with slurred speech, blurry vision, off-balance feeling, and possible left-sided mild internuclear ophthalmoplegia. Magnetic resonance imaging showed findings typical for primary demyelinating disease. Despite 5-day high-dose intravenous methylprednisolone treatment, the patient's symptoms worsened, including right-sided facial weakness, gait instability that required unilateral support, drooling, and new dorsal pontine white matter lesion on magnetic resonance imaging. Treatment with ACTH gel $80 \mathrm{U}$ for 5 consecutive days resulted in patient functional improvement, including vision and gait. ACTH gel treatment stabilized disease progression, allowing the initiation of long-term diseasemodifying treatment with monthly intravenous natalizumab. Effects of melanocortin signaling on immune function and inflammation beyond steroidogenesis provide a basis for understanding the clinical experience with ACTH gel treatment in patients with MS exacerbation.

Keywords: multiple sclerosis exacerbation, relapsing-remitting multiple sclerosis, treatment, adrenocorticotropic hormone gel

\section{Introduction}

Multiple sclerosis (MS) is a chronic demyelinating and degenerative autoimmune disease of the central nervous system (CNS) resulting from genetic and environmental risk factors. Proposed etiologies include autoimmunity against the CNS, an infectious agent, and a primary degenerative process targeting oligodendrocytes. ${ }^{1}$ Relapsing-remitting MS (RRMS), the most common subtype, involves episodes of clinical symptom exacerbation separated by periods of full or partial recovery. A relapse is defined as a sustained new or worsening neurologic symptom, in the absence of fever or infection, lasting greater than 24 hours and potentially lasting for days to weeks or even months at a time. ${ }^{2,3}$ Effective relapse treatment remains a challenge, yet is critical as patients' level of disability may continue to increase rather than return to baseline following each relapse. ${ }^{4}$ It is believed that by limiting the severity and frequency of relapses over time, one may also prevent severe disability and disease progression over time. ${ }^{3}$

To treat the acute disability seen in RRMS as well as hasten recovery from a relapse, MS practitioners have long used corticosteroid therapy. The most common treatment is a 3- to 5-day course of corticosteroids, primarily intravenous (IV) methylprednisolone (MP) 
or dexamethasone, with or without an oral steroid taper over 1 to 2 weeks. ${ }^{5}$ High-dose oral prednisone also is sometimes used. These medications can cause deleterious side effects that may limit their use, including stomach irritation, elevation of blood sugar, water retention, restlessness, insomnia, and mood swings, and long-term use is not recommended due to the potential for serious side effects, including stomach ulcers, weight gain, acne, cataracts, osteoporosis, and diabetes. Additionally, at times the patient's relapse recovery response may not be adequate following treatment with steroids. Treatment with adrenocorticotropic hormone (ACTH) gel, Food and Drug Administration (FDA)-approved to treat MS relapse, is an option for patients who are unable to tolerate the side effects of high-dose IVMP or oral prednisone, do not adequately respond to corticosteroid treatment, do not have access to IV therapy, or who have difficulty accessing veins and receiving IV medication.

ACTH gel (HP Acthar ${ }^{\circledR}$ Gel, repository corticotropin injection; Questcor Pharmaceuticals, Inc., Hayward, CA, USA) is a long-acting formulation of the full sequence $\mathrm{ACTH}_{(1-39)}$ that includes other proopiomelanocortin peptides. ${ }^{6,7}$ In 2010, the FDA reviewed and modernized the entire ACTH gel label alongside granting a new indication for infantile spasms, and following reexamination of the study data submitted in 1978, the MS exacerbation indication was reapproved for treatment of acute exacerbations in adults. ${ }^{7}$ ACTH gel has been used for several decades to treat acute exacerbations in $\mathrm{MS}^{8-12}$ and has an established safety profile. Randomized, controlled clinical trials examining ACTH gel treatment in acute exacerbations of MS (total of 298 subjects) include two placebo-controlled, double-blind studies and one double-blind comparator trial versus IVMP ${ }^{8-10}$ Early placebo-controlled studies of ACTH gel treatment of MS exacerbation demonstrated faster recovery from rapidly worsening MS compared with placebo, and the comparator study with IVMP showed equivalent, marked improvement outcomes. ${ }^{8-10}$

Interestingly, the use of corticosteroids as the common therapy to treat MS relapse is derived from these early randomized, placebo-controlled studies of ACTH treatment showing rapid improvement of relapse. ${ }^{8,9}$ The belief that the mechanisms of action and treatment effects of ACTH and high-dose corticosteroids are relatively equivalent in treating MS acute exacerbations is longstanding, with the assumption that the efficacy of ACTH is due to its corticosteroid effects on immune function and inflammation. ${ }^{10,11,13}$ However, evidence suggests ACTH may have actions independent of steroidogenesis, such as in the treatment of infantile spasms, in which ACTH has been shown effective and corticosteroid treatment has had limited effectiveness. ${ }^{14-17} \mathrm{ACTH}$ is one of several melanocortin peptides, and the emerging characterization of the melanocortin system and receptor functions beyond steroidogenesis indicates the need to reconsider ACTH in the treatment of acute MS exacerbations. ${ }^{7,18}$

The clinical case presented below describes a patient who, following nonresponse to a trial of IVMP for new-onset MS exacerbation, was effectively stabilized with ACTH gel treatment. Stabilization of the disease exacerbation then allowed the initiation of long-term disease-modifying therapy with monthly IV natalizumab.

\section{ACTH gel patient case Demographics and medical history}

The patient was a 49-year-old right-handed female who worked as a dental hygienist. She presented with a 1-day history of blurry vision, off-balance feeling, and slurred speech. She started having double vision when looking toward the left, stated that "everything seemed fuzzy," and said she felt as if she needed to hold onto something in order to keep her balance. The patient had no past medical or surgical history and no family history for MS. Diagnosis of newonset MS exacerbation followed a neurological exam and finding of cerebral spinal fluid positive for six oligoclonal bands, comprehensive metabolic and blood panels to rule out alternative diagnoses, and magnetic resonance imaging (MRI) at baseline and post-IVMP treatment consistent with McDonald 2010 criteria of dissemination of lesions in space (T2 callosal and spinal cord lesions on initial MRI) and time (new T2 dorsal pontine lesion at second MRI). ${ }^{2}$

\section{Physical and neurological exam}

The exam of cranial nerves II-XII showed possible leftsided, mild internuclear ophthalmoplegia (INO). Her gait showed slight ataxia when walking, with some difficulties with heel-toe walking after three steps. MRI testing of the patient's brain without enhancement showed findings typical for primary demyelinating disease, with several callosal plaques, right greater than left (Figure 1). No changes were seen in the brainstem or at the cervical level. MRI testing at the thoracic level showed possible demyelinating plaque at T3-T4.

\section{Treatment}

The patient was admitted to the hospital for 5 days of IVMP at 1,000 mg/day. After 3 days, she still had persistent left INO with nystagmus; however, her gait was slightly improved. 
A

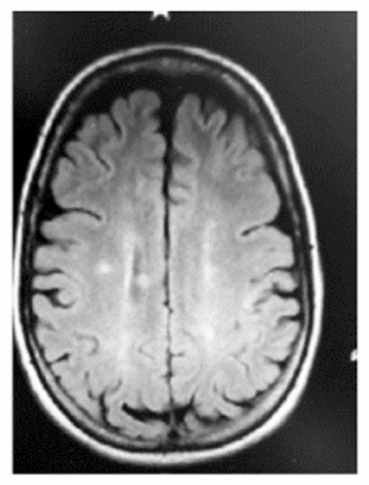

C

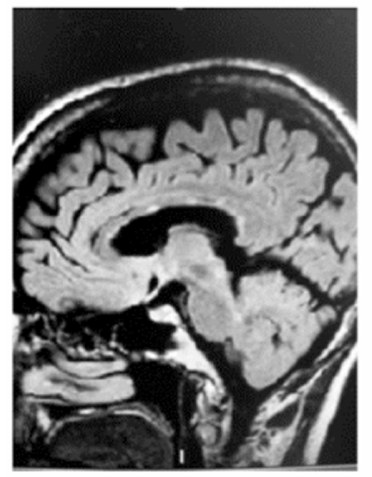

A

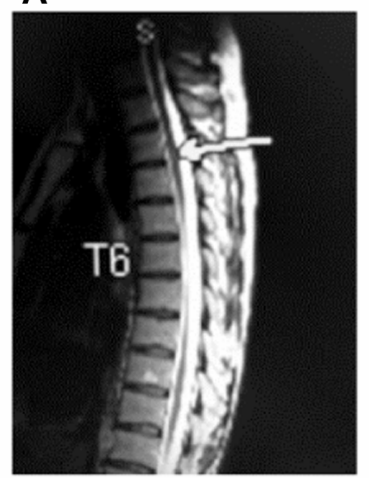

B

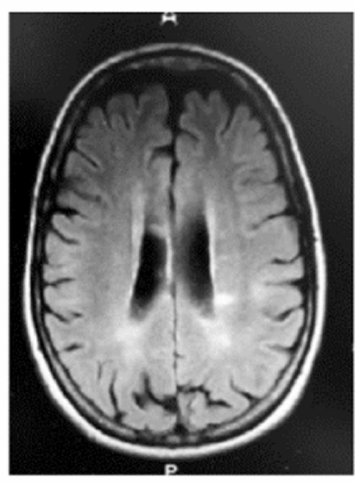

D

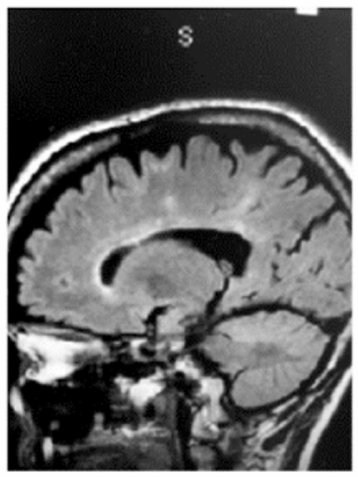

B

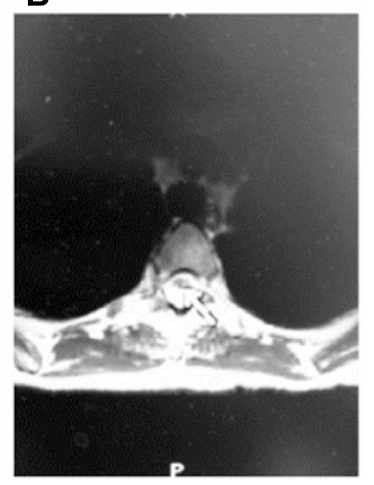

Figure I Pretreatment MRI.

Notes: (A-D, Top) Findings in brain images (images A, B FLAIR axial sequences and images C, D FLAIR sagittal sequences) are typical for primary demyelinating disease with several callosal plaques. (A-B, Bottom) Thoracic images (image A, T2 sagittal and image $\mathbf{B}$, T2 axial), suggest possible demyelinating plaque at T3-4.

Abbreviations: MRI, magnetic resonance imaging; FLAIR, fluid-attenuated inversion recovery.

The patient requested an additional 2 days of treatment as an outpatient, and she was discharged with physical therapy and neurology follow-up within 7 days.

\section{Follow-up neurological exam}

The patient was seen within 7 days of discharge, and despite 5 days of IVMP, she presented to the outpatient neurology MS center with new-onset right facial weakness. The patient also complained of drooling and gait instability. Neurologic exam

at this time showed mild bilateral INO and mild-to-moderate right facial weakness affecting the forehead, with slight Bell's phenomenon. Coordination testing showed mild finger-tonose ataxia bilaterally, and gait testing showed mild gait instability with difficulty in tandem, and equivocal toes. Herpes simplex virus evaluation was negative. The patient received an urgent MRI scan and spinal fluid analysis. The MRI revealed a new dorsal pontine white matter lesion located slightly eccentric to the right that was consistent with the clinical hypothesis of a demyelinating lesion affecting cranial nerve VII (Figure 2). The lesion did not show abnormal enhancement, but the radiologist commented that the lesion possibly showed restricted diffusion. The remainder of the brain was unchanged.

At a follow-up visit 4 days later, the patient complained of worsening symptoms of right-sided facial weakness, gait instability, and drooling. She now required assistance at times, to walk. The diagnosis given was MS exacerbation. Treatment considerations included the severity of the exacerbation, the brainstem location, and the patient's acute
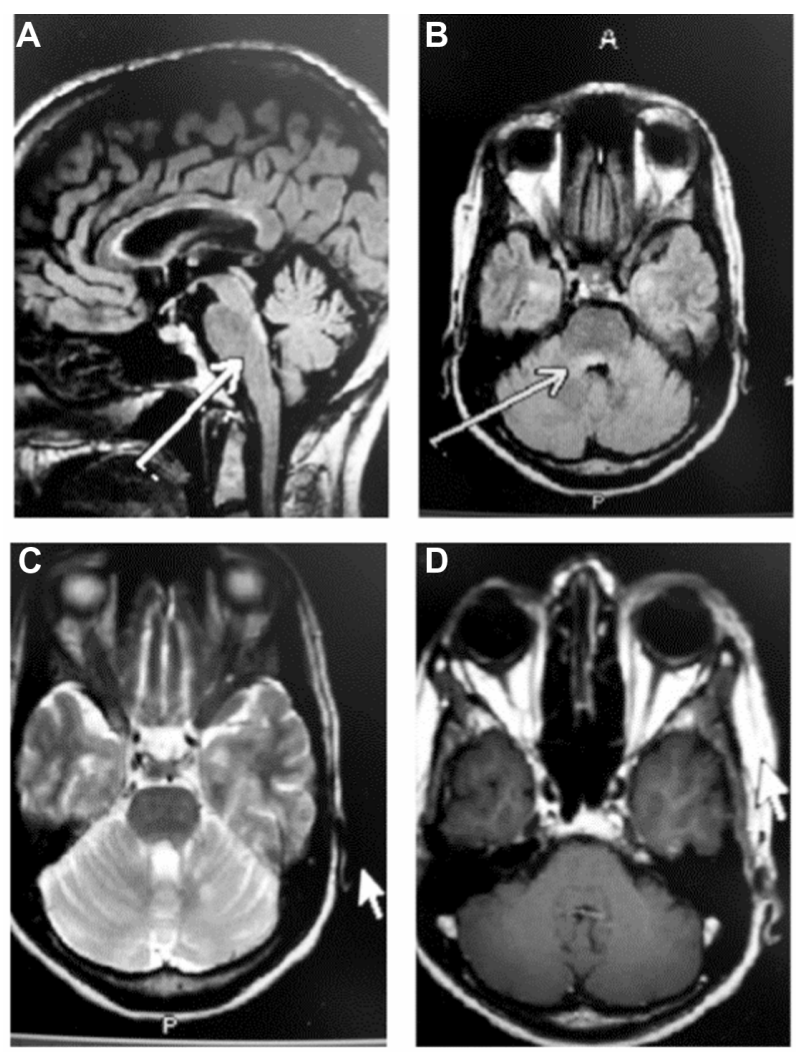

Figure 2 MRI following treatment with IVMP for 5 days.

Notes: T2 sagittal (image A) and axial (images B, C) images and TI with gadolinium (image D) show new dorsal pontine white matter lesion as depicted by the arrows in image $A$ and image $B$, located slightly eccentric to the right. Images do not show abnormal enhancement. No other changes were seen compared with pretreatment. Abbreviations: IVMP, intravenous methylprednisolone; MRI, magnetic resonance imaging. 

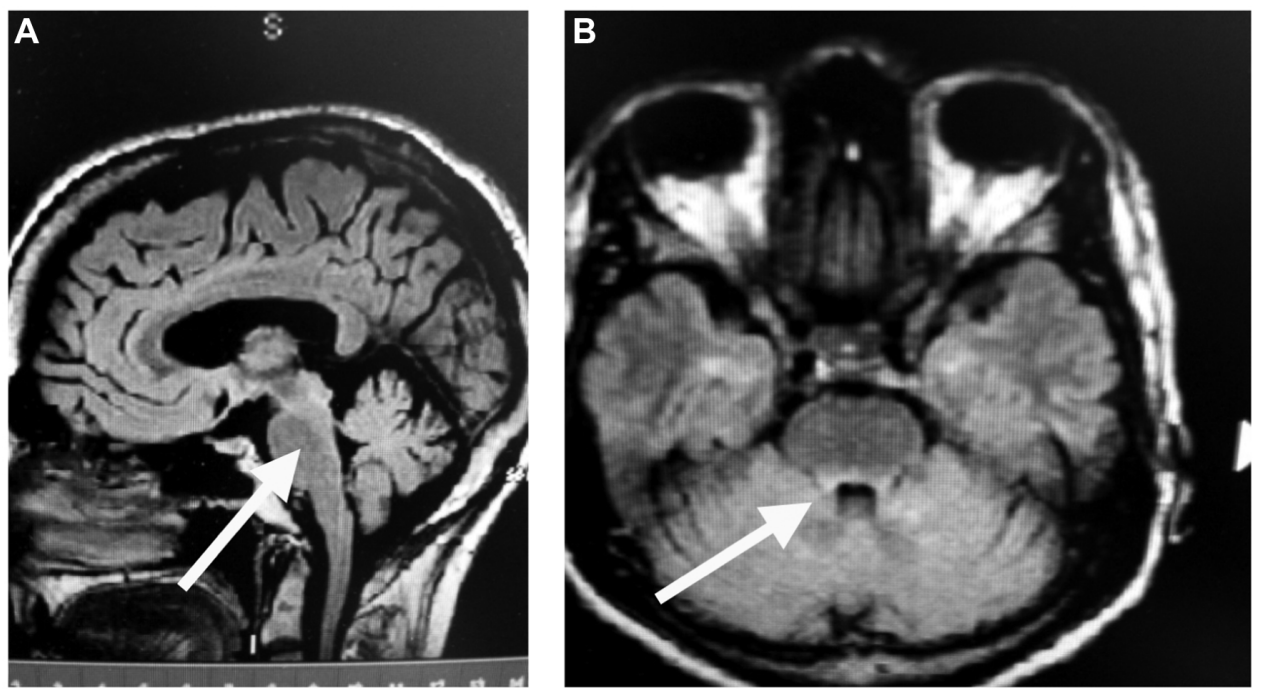

Figure 3 MRI 5 months following treatment with ACTH gel for 5 days, followed by monthly IV natalizumab.

Notes: Images are compatible with the patient's known history of demyelinating disease. The lesion in the dorsal pons, shown by the arrows in image A (FLAIR sagittal) and image B (FLAIR axial), is less prominent than was seen prior to treatment with ACTH gel and IV natalizumab.

Abbreviations: ACTH, adrenocorticotropic hormone; IV, intravenous; MRI, magnetic resonance imaging; FLAIR, fluid-attenuated inversion recovery.

worsening despite high-dose IVMP. The treatment plan options included natalizumab injection, pulse IV cyclophosphamide, plasma exchange, and ACTH gel. The treatment recommendation was to treat the patient with ACTH gel $80 \mathrm{U}$ subcutaneous injection for 5 consecutive days with the goal to rapidly stabilize disease.

At the follow-up visit 7 days later, the patient had completed her course of ACTH gel and reported improvement in her vision and gait. She reported feeling more steady on her feet but said she still required some walking assistance at times. The patient's facial weakness was no longer a complete Bell's-like phenomenon and was significantly improved. The neurologic exam at this time showed mild residual bilateral INO, improved right facial weakness with improved forehead and frontalis musculature strength, and improved asymmetry. The patient had mild finger-to-nose ataxia and some slight gait instability with some difficulty in tandem. The patient showed stabilization of disease exacerbation in response to ACTH gel, despite being an IVMP nonresponder. The patient was in the most severe form of an MS exacerbation, with worsening brainstem presentation. Treatment with ACTH gel stabilized the continued worsening of the patient's persistent disease progression and actually improved some of the patient's brainstem symptoms, such as facial weakness and eye movement weakness. The patient was subsequently started on monthly IV natalizumab, approximately 1 to 2 weeks following cessation of ACTH gel, for long-term immunemodulating MS treatment.
At 5 months follow-up, the patient had returned to baseline function and had been working for 3 months. The patient was doing very well except for some mild loss of energy, and the neurologic exam was normal. MRI testing of the brain revealed findings compatible with the patient's known history of demyelinating disease; however, the previously described lesion in the dorsal pons was less prominent (Figure 3).

\section{Conclusion}

Effects of melanocortin signaling on immune function and inflammation provide a basis for understanding the clinical experience with ACTH gel in the treatment of patients with MS relapse. ${ }^{7,18,19}$ Emerging understanding of the melanocortin system and interaction of ACTH with widespread melanocortin receptors strongly suggests that the mechanisms of action of ACTH gel in MS are not limited to steroidogenesis effects. ${ }^{20,21}$ Although no studies are currently available examining ACTH gel efficacy in patients with MS exacerbation who are refractory to steroids and other therapies, this has been shown in other disorders, including infantile spasms. ${ }^{14-17}$ Consistent with the emerging understanding of ACTH gel mechanisms of action, ACTH gel has been proposed within a relapse management treatment algorithm for MS, as a second-line treatment for patients who do not improve or are unable to tolerate steroid treatment. ${ }^{22}$ In the clinical case that was presented, the patient experienced severe progression of new-onset MS exacerbation with brainstem presentation. Despite 5 days of high-dose IVMP, the patient's symptoms actually worsened. ACTH gel stabilized the progressive 
course of the MS exacerbation, was associated with some improvement in vision and gait, and provided valuable time needed to initiate long-term disease-modifying therapy with natalizumab. Patients with RRMS who show nonresponse to conventional IV steroid formulations, or who are unable to tolerate associated side effects, are an important subgroup of patients for consideration of treatment with ACTH gel. Thus, the awareness of a treatment alternative such as ACTH gel, with demonstrated efficacy and suggested mechanisms of action beyond steroidogenesis, is important.

\section{Acknowledgment}

The author thanks Lynanne McGuire, PhD, of MedVal Scientific Information Services, LLC, for providing medical writing and editorial assistance. This manuscript was prepared according to the International Society for Medical Publication Professionals" "Good Publication Practice for Communicating Company-Sponsored Medical Research: The GPP2 Guidelines."

Funding to support preparation of this manuscript was provided by Questcor Pharmaceuticals.

\section{Disclosure}

SN has had speaking and consultancy relationships with the following entities: Questcor Pharmaceuticals, Teva Neuroscience, Biogen, Pfizer, Accorda, Serono, and Novartis. The author reports no other conflicts of interest.

\section{References}

1. Boppana S, Huang H, Ito K, Dhib-Jalbut S. Immunologic aspects of multiple sclerosis. Mt Sinai J Med. 2011;78(2):207-220.

2. Polman $\mathrm{CH}$, Reingold SC, Banwell B, et al. Diagnostic criteria for multiple sclerosis: 2010 revisions to the McDonald criteria. Ann Neurol. 2011;69(2):292-302.

3. Vollmer T. The natural history of relapses in multiple sclerosis. J Neurol Sci. 2007;256(Supp1 1):S5-S13.

4. Lublin FD, Baier M, Cutter G. Effect of relapses on development of residual deficit in multiple sclerosis. Neurology. 2003;61(11): 1528-1532.

5. National Clinical Advisory Board (National Multiple Sclerosis Society). Recommendations Regarding Corticosteroids in the Management of Multiple Sclerosis. New York, NY: National Multiple Sclerosis Society; 2008.

6. HP Acthar ${ }^{\circledR}$ Gel (repository corticotropin) injection [prescribing information]. Hayward, CA: Questcor Pharmaceuticals, Inc.; 2012.

International Medical Case Reports Journal

\section{Publish your work in this journal}

The International Medical Case Reports Journal is an international, peer-reviewed open-access journal publishing original case reports from all medical specialties. Previously unpublished medical posters are also accepted relating to any area of clinical or preclinical science. Submissions should not normally exceed 2,000 words or
7. Arnason BG, Berkovich R, Catania A, Lisak RP, Zaidi M. Mechanisms of action of adrenocorticotropic hormone and other melanocortins relevant to the clinical management of patients with multiple sclerosis. Mult Scler. 2013;19(2):130-136.

8. Miller H, Newell DJ, Ridley A. Multiple sclerosis. Treatment of acute exacerbations with corticotrophin (ACTH). Lancet. 1961; 278(7212):1120-1122.

9. Rose AS, Kuzma JW, Kurtzke JF, Namerow NS, Sibley WA, Tourtellotte WW. Cooperative study in the evaluation of therapy in multiple sclerosis. ACTH vs placebo - final report. Neurology. 1970; 20(5):1-59.

10. Thompson AJ, Kennard C, Swash M, et al. Relative efficacy of intravenous methylprednisolone and ACTH in the treatment of acute relapse in MS. Neurology. 1989;39(7):969-971.

11. Filippini G, Brusaferri F, Sibley WA, et al. Corticosteroids or ACTH for acute exacerbations in multiple sclerosis. Cochrane Database Syst Rev. 2000:CD001331.

12. Simsarian JP, Saunders C, Smith DM. Five-day regimen of intramuscular or subcutaneous self-administered adrenocorticotropic hormone gel for acute exacerbations of multiple sclerosis: a prospective, randomized, open-label pilot trial. Drug Des Devel Ther. 2011;5:381-389.

13. Sibley WA. Spotlight series: pivotal trials through today's knowledge adrenocorticotrophic hormone. Int MS J. 2009;16(2):42-46.

14. Stafstrom CE, Arnason BG, Baram TZ, et al. Treatment of infantile spasms: emerging insights from clinical and basic science perspectives. J Child Neurol. 2011;26(11):1411-1421.

15. Baram TZ, Mitchell WG, Tournay A, Snead OC, Hanson RA, Horton EJ. High-dose corticotropin (ACTH) versus prednisone for infantile spasms: a prospective, randomized, blinded study. Pediatrics. 1996;97(3):375-379.

16. Mackay MT, Weiss SK, Adams-Webber T, et al; American Academy of Neurology; Child Neurology Society. Practice parameter: medical treatment of infantile spasms: report of the American Academy of Neurology and the Child Neurology Society. Neurology. 2004;62(10): 1668-1681.

17. Go CY, Mackay MT, Weiss SK, et al; Child Neurology Society; American Academy of Neurology. Evidence-based guideline update: medical treatment of infantile spasms. Report of the Guideline Development Subcommittee of the American Academy of Neurology and the Practice Committee of the Child Neurology Society. Neurology. 2012;78(24):1974-1980.

18. Ross AP, Ben-Zacharia A, Harris C, Smrtka J. Multiple sclerosis, relapses, and the mechanism of action of adrenocorticotropic hormone. Front Neurol. 2013;4(21):1-12.

19. Catania A, Gatti S, Colombo G, Lipton JM. Targeting melanocortin receptors as a novel strategy to control inflammation. Pharmacol Rev. 2004;56(1):1-29.

20. Luger TA, Brzoska T. alpha-MSH related peptides: a new class of anti-inflammatory and immunomodulating drugs. Ann Rheum Dis. 2007;66 Suppl 3:iii52-iii55.

21. Brzoska T, Luger TA, Maaser C, Abels C, Böhm M. Alpha-melanocytestimulating hormone and related tripeptides: biochemistry, antiinflammatory and protective effects in vitro and in vivo, and future perspectives for the treatment of immune-mediated inflammatory diseases. Endocr Rev. 2008;29(5):581-602.

22. Berkovich R. Treatment of acute relapses in multiple sclerosis. Neurotherapeutics. 2013;10(1):97-105.

\section{Dovepress}

4 published pages including figures, diagrams and references. The manuscript management system is completely online and includes a very quick and fair peer-review system, which is all easy to use. Visit $\mathrm{http}: / / \mathrm{ww}$ w.dovepress.com/testimonials.php to read real quotes from published authors. 